\title{
Calibration-Free Pulse Oximetry Based on Two Wavelengths in the Infrared - A Preliminary Study
}

\section{Meir Nitzan ${ }^{1}{ }^{*}$, Salman Noach ${ }^{1}$, Elias Tobal ${ }^{1}$, Yair Adar ${ }^{1}$, Yaacov Miller ${ }^{1}$, Eran Shalom ${ }^{1}$ and Shlomo Engelberg ${ }^{2}$}

1 Department of Applied Physics/Medical Engineering, Jerusalem College of Technology, Jerusalem 9116001, Israel; E-Mails: salman@jct.ac.il (S.N.); elitobal@gmail.com (E.T.); yadar@g.jct.ac.il (Y.A.); yalamiller@gmail.com (Y.M.); eransh@g.jct.ac.il (E.S.)

2 Department of Electronics, Jerusalem College of Technology, Jerusalem 9116001, Israel; E-Mail: shlomoe@jct.ac.il

* Author to whom correspondence should be addressed; E-Mail: nitzan@ @ jct.ac.il; Tel.: +972-2-675-1139; Fax: +972-2-675-1045.

Received: 23 January 2014; in revised form: 9 April 2014 / Accepted: 14 April 2014 /

Published: 23 April 2014

\begin{abstract}
The assessment of oxygen saturation in arterial blood by pulse oximetry $\left(\mathrm{SpO}_{2}\right)$ is based on the different light absorption spectra for oxygenated and deoxygenated hemoglobin and the analysis of photoplethysmographic (PPG) signals acquired at two wavelengths. Commercial pulse oximeters use two wavelengths in the red and infrared regions which have different pathlengths and the relationship between the PPG-derived parameters and oxygen saturation in arterial blood is determined by means of an empirical calibration. This calibration results in an inherent error, and pulse oximetry thus has an error of about $4 \%$, which is too high for some clinical problems. We present calibration-free pulse oximetry for measurement of $\mathrm{SpO}_{2}$, based on PPG pulses of two nearby wavelengths in the infrared. By neglecting the difference between the path-lengths of the two nearby wavelengths, $\mathrm{SpO}_{2}$ can be derived from the PPG parameters with no need for calibration. In the current study we used three laser diodes of wavelengths 780, 785 and $808 \mathrm{~nm}$, with narrow spectral line-width. $\mathrm{SaO}_{2}$ was calculated by using each pair of PPG signals selected from the three wavelengths. In measurements on healthy subjects, $\mathrm{SpO}_{2}$ values, obtained by the 780-808 nm wavelength pair were found to be in the normal range. The measurement of $\mathrm{SpO}_{2}$ by two nearby wavelengths in the infrared with narrow line-width enables the assessment of $\mathrm{SpO}_{2}$ without calibration.
\end{abstract}


Keywords: oxygen saturation; pulse oximetry; infrared; arterial blood; diode lasers; calibration

\section{Introducation}

\subsection{Pulse Oximetry for the Measurement of Arterial Oxygen Saturation}

The transfer of oxygen from the lungs to the tissue cells is largely carried out by the hemoglobin molecules in the red blood cells and only $2 \%$ of the total oxygen content is dissolved in the plasma. Oxygen saturation in the blood is the ratio of oxygenated hemoglobin concentration to total hemoglobin concentration in the blood, and its value in the arterial blood, $\mathrm{SaO}_{2}$, is of great clinical and physiological significance since it reflects the adequacy of oxygen delivery and respiratory function. Normal values of $\mathrm{SaO}_{2}$ are $94 \%-98 \%$ at sea level but the values may decline somewhat beyond the age of 70 years [1].

$\mathrm{SaO}_{2}$ can be assessed in vitro, in extracted arterial blood, either directly by means of co-oximetry or by measuring oxygen partial pressure and using the oxygen-hemoglobin dissociation curve. Estimation of $\mathrm{SaO}_{2}$ can also be obtained non-invasively by pulse oximetry [2-4], which is based on the different light absorption spectra for oxygenated and de-oxygenated hemoglobin (Figure 1). In order to assess $\mathrm{SaO}_{2}$, the contribution of the arterial blood to the light absorption must be isolated from that of the venous blood, and in pulse oximetry it is achieved by photoplethysmography (PPG)-the measurement of light absorption changes due to the cardiac-induced blood volume changes. The PPG probe consists of a light source emitting light into the tissue and a detector measuring the intensity of light transmitted through the tissue, which decreases during systole because of the systolic increase in the arterial blood volume (Figure 2). Since the PPG pulse represents light absorption in arterial blood, PPG signals at two wavelengths enable the assessment of oxygen saturation in the arterial blood [2]. As will be explained below, there is still a discrepancy between the value of arterial oxygen saturation obtained by pulse oximetry and that obtained by direct measurements in blood extracted from the arteries, and it is therefore customary to designate the former by $\mathrm{SpO}_{2}$ while the latter retains the name $\mathrm{SaO}_{2}$. [4-6].

Figure 1. Molar extinction coefficients of oxygenated $\left(\mathrm{HbO}_{2}\right)$ and deoxygenated $(\mathrm{Hb})$ hemoglobin as a function of the wavelength. Prepared by Dr. Scott Prahl from a variety of sources [7]. Used with permission from Dr. Prahl.

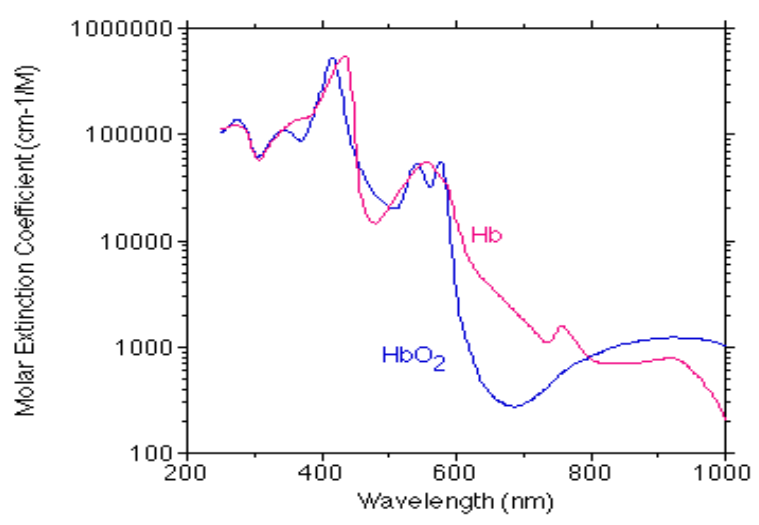


Figure 2. The PPG pulse. The transmitted light through the tissue decreases during systole and increases during diastole. $\mathrm{I}_{\mathrm{D}}$ and $\mathrm{I}_{\mathrm{S}}$ represent the maximal and minimal light transmission through the tissue.

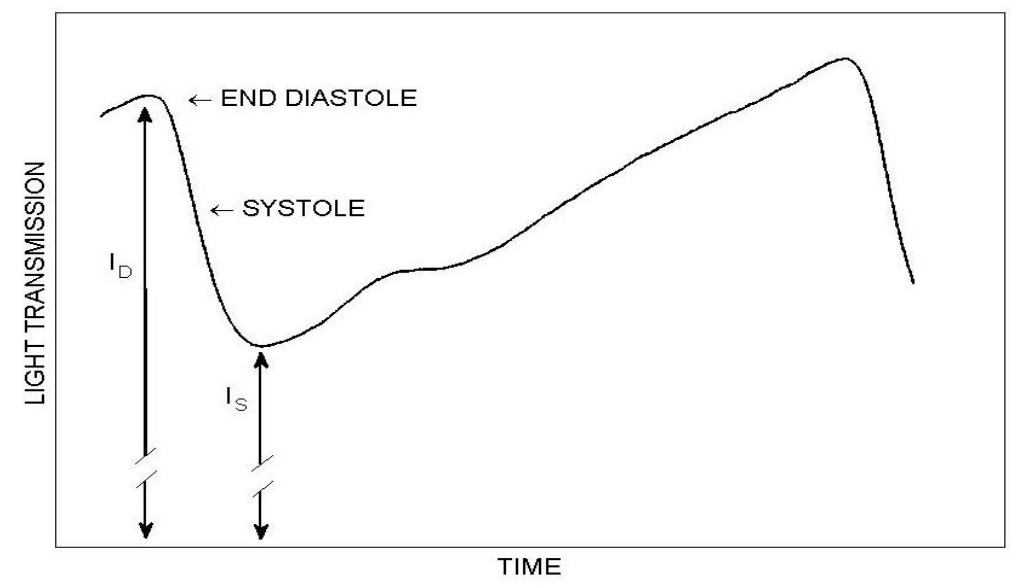

The theory of conventional pulse oximetry has been described in several publications [3,8-10]. PPG curves in two wavelengths are recorded and $\mathrm{SpO}_{2}$ is derived from the ratio of ratios $\mathrm{R}$ which is defined by:

$$
\mathrm{R}=\frac{(\mathrm{AC} / \mathrm{DC})_{1}}{(\mathrm{AC} / \mathrm{DC})_{2}}
$$

where AC and DC are the peak-to-peak amplitude and the baseline of the PPG pulse, respectively. In commercial pulse oximeters the two wavelengths are chosen in the red and infrared regions, where the difference in light absorption between the two wavelengths is relatively large. However, the transmitted light intensity through a tissue sample which includes blood vessels is also affected by light scattering in the tissue and in the blood. For the choice of two wavelengths in the red and infrared the scattering coefficient and the optical path-length differ significantly between the two wavelengths $[3,9,11,12]$ and the relationship between the physiological parameter, $\mathrm{SaO}_{2}$, and the measured parameter $\mathrm{R}$ cannot be derived directly from physical and physiological considerations of light absorption in oxygenated and deoxygenated hemoglobin, using the Lambert-Beer Law. The relationship between $\mathrm{R}$ and $\mathrm{SaO}_{2}$ is found experimentally for each type of commercial pulse oximeter sensor by calibration [3,4]: $\mathrm{R}$ is measured in several persons simultaneously with in vitro $\mathrm{SaO}_{2}$ measurement in extracted arterial blood by means of a co-oximeter. The formula relating $\mathrm{R}$ to oxygen saturation measured by pulse oximetry, $\mathrm{SpO}_{2}$, is then determined by proposing a mathematical relationship, such as:

$$
\mathrm{SpO}_{2}=\frac{\mathrm{k}_{1}-\mathrm{k}_{2} \mathrm{R}}{\mathrm{k}_{3}-\mathrm{k}_{4} \mathrm{R}}
$$

and obtaining the values of the constants $\mathrm{k}_{\mathrm{i}}$ for the specific pulse oximeter by best fit analysis of the measured parameters in the calibration process.

An analytical relationship between $\mathrm{R}$ and $\mathrm{SpO}_{2}$ can be obtained by making use of the Lambert-Beer Law and analysis of the light absorption and scattering in tissue which includes blood with oxygenated 
and deoxygenated hemoglobin $[9,11]$. The following relationship between the ratio $\mathrm{R}$ and the oxygen saturation $\mathrm{SpO}_{2}$ is then derived:

$$
\mathrm{SpO}_{2}=\frac{\varepsilon_{\mathrm{d} 1}-\mathrm{R}\left(\mathrm{l}_{2} / 1_{1}\right) \varepsilon_{\mathrm{d} 2}}{\mathrm{R}\left(\mathrm{l}_{2} / 1_{1}\right)\left(\varepsilon_{02}-\varepsilon_{\mathrm{d} 2}\right)+\left(\varepsilon_{\mathrm{d} 1}-\varepsilon_{01}\right)}
$$

where $\varepsilon_{\mathrm{o}}$ and $\varepsilon_{\mathrm{d}}$ are the extinction coefficients for oxygenated and deoxygenated hemoglobin, respectively (the extinction coefficient of hemoglobin is defined as the absorption constant of the sample divided by the hemoglobin concentration). The constants $l_{1}$ and $l_{2}$ are the path-lengths for the two wavelengths and depend strongly on the scattering coefficient.

For the two wavelengths in the red and infrared regions which are used by the common commercial pulse oximeters, $1_{1}$ and $l_{2}$ are expected to differ and they are unknown. $\mathrm{SpO}_{2}$ can be derived from $\mathrm{R}$ through the calibration process by assuming that $l_{2} / l_{1}$ is a constant that is independent of inter-subject variability in the circulatory system. In this case the coefficients of R in Equation (3) are constants and can be determined through calibration, as described above [5,11]. If the parameter $l_{2} / l_{1}$ changes between different subjects, in particular between the healthy subjects on whose fingers the calibration was performed and the patients on whose fingers the clinical examination is carried out, inaccuracy in $\mathrm{SpO}_{2}$ measurement is to be expected.

\subsection{Accuracy of Pulse Oximetry}

Most manufacturers of pulse oximeters claim an accuracy of $2 \%[13,14]$, which is the standard deviation (SD) of the differences between $\mathrm{SpO}_{2}$ and $\mathrm{SaO}_{2}$ measured on extracted arterial blood samples by a co-oximeter. An SD of $2 \%$ means that an error of $4 \%$ or more (2SD) is expected in $5 \%$ of the examinations, assuming that the differences between $\mathrm{SpO}_{2}$ and $\mathrm{SaO}_{2}$ are normally distributed. Since the full range of $\mathrm{SaO}_{2}$ in adults, even including most of the sick patients is $80 \%-100 \%$, an error of $3 \%-4 \%$ may be significant.

Despite its potential error of $3 \%-4 \%$, pulse oximetry is a clinically valuable tool for monitoring patients during surgery or rehabilitation, ensuring intraoperative and post-operative patient safety. Pulse oximetry can detect a sudden decrease of $\mathrm{SpO}_{2}$ by $3 \%-4 \%$, enabling the early detection of acute deterioration of the respiratory function of the patient or technical failure of mechanical ventilatory support and is, therefore, considered a mandatory tool for most patients in operating rooms and intensive care units.

An inaccuracy of $3 \%-4 \%$ in oxygen saturation measurement is, however, too high to allow such pulse oximeters to be used in routine management of various patient populations, in particular critically ill patients. Because of the low accuracy of $\mathrm{SpO}_{2}$ measurements, some researchers suggested that $\mathrm{SpO}_{2}$ levels as high as $94 \%$ or $96 \%$ should be maintained, in order to ensure $\mathrm{SaO}_{2}$ value of $90 \%$ during mechanical ventilation and oxygen support $[1,15,16]$. In a study on critically ill patients, relatively low correlation was found between spontaneous changes in $\mathrm{SpO}_{2}$ and in $\mathrm{SaO}_{2}$ $\left(\mathrm{r}=0.6, \mathrm{r}^{2}=0.37\right)$ [17], and the authors inferred that changes in $\mathrm{SpO}_{2}$ do not reliably predict equivalent changes in $\mathrm{SaO}_{2}$ in the critically ill. In a study on patients with severe sepsis and septic shock in emergency department, the mean difference between $\mathrm{SpO}_{2}$ and $\mathrm{SaO}_{2}$ was $2.75 \%$ and the standard deviation $3.1 \%$ [18]. 
The low accuracy of pulse oximetry can be attributed, at least partly, to the calibration process currently used for pulse oximeters, which is based on examinations on healthy volunteers and is not necessarily applicable to sick patients, in particular neonates [14]. Since the calibration process is based on $\mathrm{SaO}_{2}$ measurements by co-oximeter, the inaccuracy in the latter also contributes to the error in $\mathrm{SpO}_{2}$ measurement [13]. The discrepancy between $\mathrm{SpO}_{2}$ and $\mathrm{SaO}_{2}$ is greater at saturations below 75\%-85\% [1,10,18-20], because ethical restrictions prevent manufacturers from reducing $\mathrm{SaO}_{2}$ below $80 \%$ during the calibration process.

\subsection{A Calibration-Free Pulse Oximeter}

If the two wavelengths are sufficiently close so that the difference between their path-lengths can be neglected $\left(1_{2} / l_{1} \approx 1\right)$, the relationship between the ratio $\mathrm{R}$ and $\mathrm{SpO}_{2}$ becomes $[3,8,21]$ :

$$
\mathrm{SpO}_{2}=\frac{\varepsilon_{\mathrm{d} 1}-\mathrm{R} \varepsilon_{\mathrm{d} 2}}{\mathrm{R}\left(\varepsilon_{02}-\varepsilon_{\mathrm{d} 2}\right)+\left(\varepsilon_{\mathrm{d} 1}-\varepsilon_{01}\right)}
$$

Equation (4) enables the calculation of $\mathrm{SpO}_{2}$ from the measured parameter $\mathrm{R}$ and the values of the extinction coefficients with no need for calibration. This was shown by Nitzan et al. [21], using two infrared light emitting diodes (LEDs) with emission spectra that peaked at wavelengths 767 and 811 $\mathrm{nm}$. The $\mathrm{SpO}_{2}$ values, found using Equation (4), were in the range of $90 \%-100 \%$, while $\mathrm{SpO}_{2}$ values obtained by commercial pulse oximeters (using red and infrared light and calibration) were 96\%-98\%. In that study, the values of the hemoglobin extinction coefficients in Equation (4) were taken as the mean values of the corresponding values presented in several data bases, averaged over the emission spectra of the LEDs.

The low accuracy achieved by the calibration-free pulse oximetry in that study [21] can be attributed, at least partly, to the broad emission spectra of the LEDs resulting in inaccuracy in the determination of the mean extinction coefficient. In the current study, the LEDs, have been replaced by infrared laser diodes. The narrow emission spectra of the laser diodes allow the use of specific values of the extinction coefficients for hemoglobin. Additionally, as described in the next section, the analysis of the PPG pulses was improved.

\section{Experimental Section}

\subsection{Subjects and Methods}

$\mathrm{SpO}_{2}$ was measured on 15 male subjects aged 20-35 years. Each finger temperature was above $30{ }^{\circ} \mathrm{C}$. For each subject PPG signals in three wavelengths, 780, 785 and $808 \mathrm{~nm}$, were recorded for about $90 \mathrm{~s}$, and $\mathrm{SpO}_{2}$ was derived from each pair of wavelengths as described below. Simultaneous $\mathrm{SaO}_{2}$ measurements were also performed on these subjects using a commercial pulse oximeter (BCI 3301 Smiths Medical PM, Waukesha, WI, USA) for comparison.

\subsection{The Optical System}

When using Equation (4) to determine the value of $\mathrm{SpO}_{2}$, it is important to ensure that the wavelength of the light used to measure the PPG is stable. An error of $\pm 0.2 \mathrm{~nm}$ in the wavelength 
causes an error in the determination of the extinction coefficient and leads to an error of about $\pm 0.5 \%$ in $\mathrm{SaO}_{2}$ for the $780 \mathrm{~nm}-808 \mathrm{~nm}$ and $785 \mathrm{~nm}-808 \mathrm{~nm}$ pairs and much more for the $780 \mathrm{~nm}-785 \mathrm{~nm}$ pair. Hence, a well-defined, stable wavelength is needed for the calibration-free pulse oximetry.

In the current study we used three wavelength-stabilized laser diodes (Innovative Photonic Solutions, Inc. Monmouth Junction, NJ, USA) of wavelengths 780, 785 and $808 \mathrm{~nm}$, with narrow spectral bandwidth (less than $0.15 \mathrm{~nm}$ ). For each diode, the manufacturer's data sheets were used to determine a temperature range over which the wavelength dependence on temperature is small. Thermoelectric coolers (TECs) (TCLDM9 Thorlabs, Newton, NJ, USA) that make use of the Peltier effect were used to stabilize the temperature (mainly in order to stabilize the light intensity). Changes in the TEC temperature by $\pm 3{ }^{\circ} \mathrm{C}$ and changes in the diode current in the range 80-140 $\mathrm{mA}$ changed the wavelength by less than $0.1 \mathrm{~nm}$ (measured by an Agilent 86140, optical spectral analyzer, Santa Rosa, CA, USA).

The light emitted from each of the three light sources needs to be delivered to the same site in the finger in order to illuminate the same blood volume for each pair of wavelengths. The beams from the three laser diodes were merged randomly and delivered to the same measurement site by using a trifurcated fiber bundle (Oriel-Newport Model 77536, Irvine, CA, USA) which was comprised of three input bundles (each with a diameter of $3.2 \mathrm{~mm}$ ) and a common bundle (with a diameter of $5.5 \mathrm{~mm}$ ) (Figure 3). The latter was comb randomized, so the output light from each input bundle was evenly divided in the common bundle.

Figure 3. Schematics of the optical setup. The three laser diodes were coupled to a trifurcated fiber bundle. The common bundle was attached to the finger probe parallel to the finger surface. The reflecting prism is opposite to the detector.

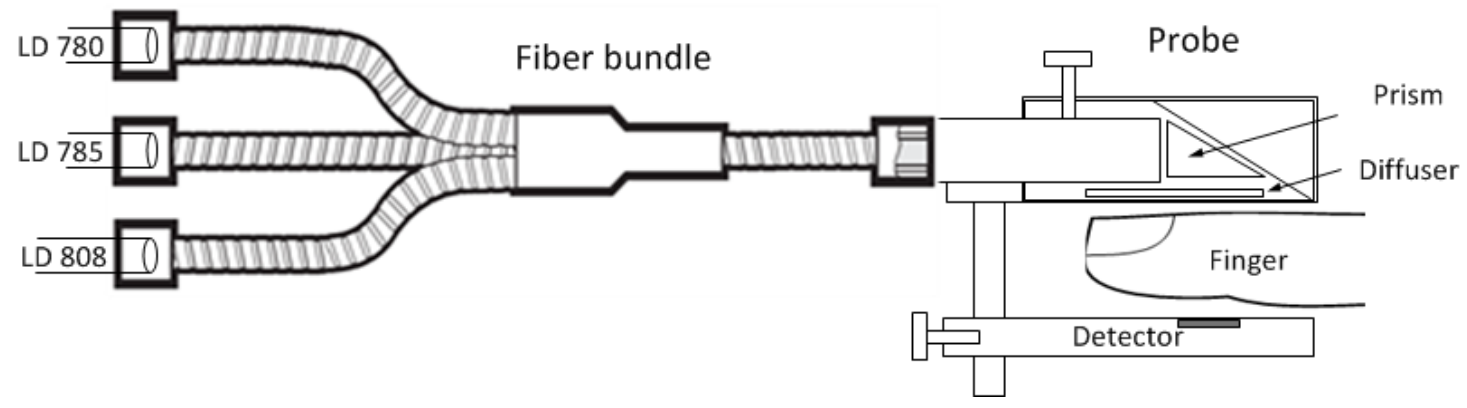

The finger transmission probe (Figure 3) had an input port for the common fiber bundle end and a PIN photodiode (BPW 34, Vishay Electronics, Malvern, PA, USA, $5.4 \times 4.3 \mathrm{~mm}^{2}$ ) placed opposite the fiber bundle end. The light was reflected by $90^{\circ}$ through a prism inside the probe, allowing the fiber bundle to be attached parallel to the finger, for higher mechanical stability. A diffuser (Light Shaping Diffuser, Luminit, Torrance, CA, USA) was placed after the prism to homogenize the light distribution and to reduce speckle noise. A photograph of the probe is shown in Figure 4.

\subsection{The Electronic System}

A block diagram of the measurement system is given in Figure 5. Light from the laser diodes is emitted sequentially, by time sharing, using driver circuits controlled by a microcontroller. The light 
transmitted through the finger is detected by the photodetector, whose output is digitized and processed by the microcontroller and conveyed to a PC for offline analysis. The temperature of each TEC is determined via the PC. The power to the PPG device was supplied by three $1.5 \mathrm{~V}$ AA batteries.

Figure 4. Photograph of the finger probe. Light is delivered to one side of the finger and the transmitted light is collected by the detector.

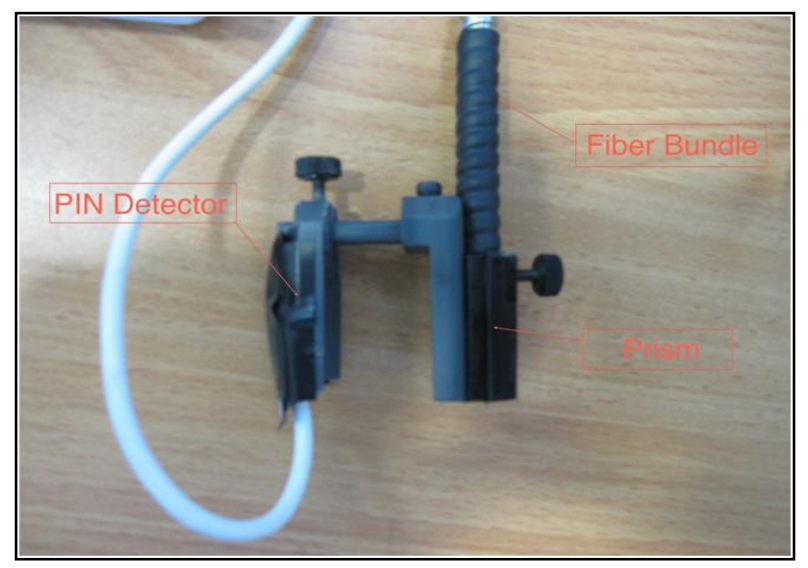

Figure 5. Block diagram of the measurement system.

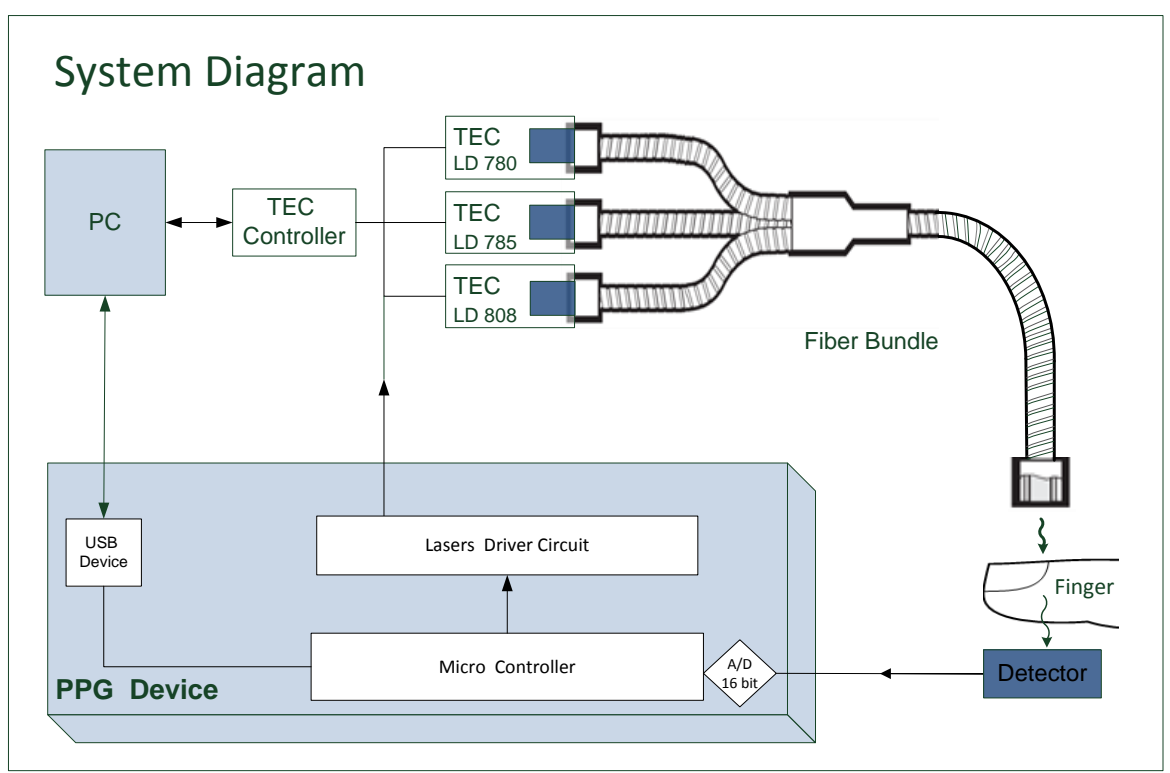

The PPG signal for each wavelength was separated by means of time sharing between the laser diodes. During each cycle of $2,556 \mu$ s, each laser diode was turned on for $511.2 \mu$ s and off for the rest of the cycle. Each of the three wavelengths and the dark current were sampled 391.22 times per second (in order to achieve good temporal resolution for the PPG pulses at the three wavelengths and to avoid aliasing of $50 \mathrm{~Hz}$ noise). Consequently, the detector output was sampled at $1564.88 \mathrm{~Hz}$ and digitized using a 16-bit A/D.

The first sample of the detector output for each laser diode was taken $383.4 \mu$ s after turning on the laser diode. This was done in order to stabilize the laser diode, so that the actual sampling was carried out for a period of $127.8 \mu \mathrm{s}$ (Figure 6). During this interval, 25 readings were acquired by the A/D and averaged by the microcontroller. Then a dark period of $127.8 \mu$ s was used to avoid cross-talk between 
channels. The second and third laser diodes were pulsed in the same sequence, and afterwards the dark current was sampled. Figure 6 shows a diagram of the time division. The signal was exported to MATLAB for post-processing and the system had the ability to display the signal in real time on the PC.

Figure 6. Schematic presentation of the time division of the laser diode activation between the three diodes.

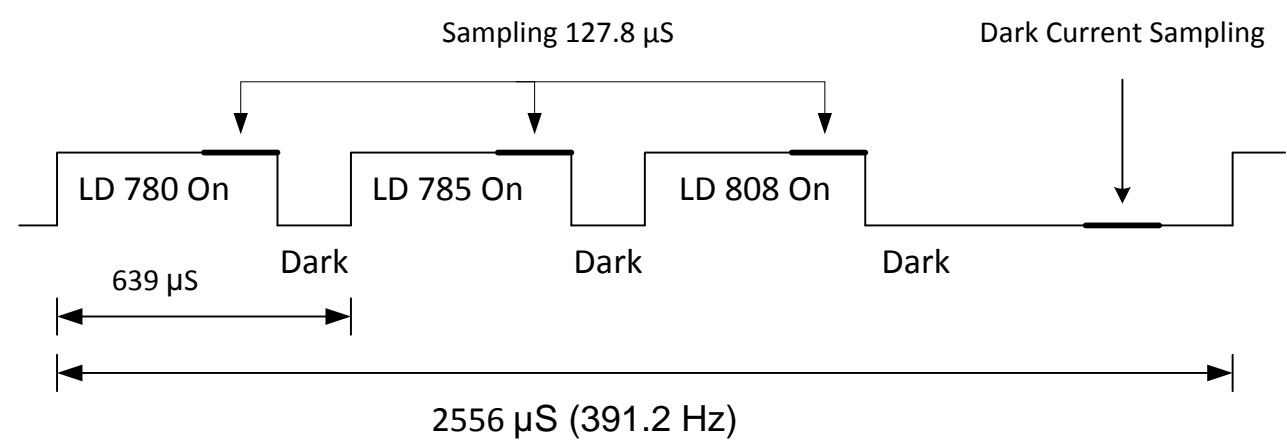

\subsection{Determination of $\mathrm{SpO}_{2}$}

$\mathrm{SpO}_{2}$ was determined by using Equation (4). The analytical derivation of Equation (4) from the Beer-Lambert law [11,21] shows that in order to obtain $\mathrm{SpO}_{2}$ through Equation (4) the ratio of ratios, $\mathrm{R}$, should be calculated as follows:

$$
R=\frac{\left[\left(I_{D}-I_{S}\right) / I_{S}\right]_{1}}{\left[\left(I_{D}-I_{S}\right) / I_{S}\right]_{2}}
$$

where $I_{D}$ is the value of the PPG pulse at end-diastole and $I_{S}$ is the minimum value of the PPG pulse during systole (see Figure 2). Note that the value of DC in Equation (1) was replaced by $I_{S}$. Due to the slow trend of the baseline $I_{D}$ of the PPG pulse, the value of $I_{D}$ (for the determination of the pulse amplitude, $\mathrm{I}_{\mathrm{D}}-\mathrm{I}_{\mathrm{S}}$ ) was taken as the value of the extrapolated line connecting the two maxima of the pulse at the time of pulse minimum, as shown in Figure 7. The pulse amplitude was taken as the difference between the extrapolated value of $I_{D}$ and the measured value of $I_{S}$. For each wavelength, the pulse amplitude and $\mathrm{I}_{\mathrm{S}}$ were derived for each of twenty pulses; for each pair of wavelengths the $\mathrm{R}$ value was calculated for each pulse by using Equation (5); then the mean value of $R$ for the twenty pulses was taken as the $\mathrm{R}$ value for this pair of wavelengths.

In order to use Equation (4) to derive $\mathrm{SaO}_{2}$ the values of $\varepsilon_{\mathrm{o}}$ and $\varepsilon_{\mathrm{d}}$, the extinction coefficient values for oxygenated $\left(\mathrm{HbO}_{2}\right)$ and deoxygenated $(\mathrm{Hb})$ hemoglobin, are required for each wavelength. The absorption spectra for $\mathrm{Hb}$ and $\mathrm{HbO}_{2}$ have been measured and reported by several research groups [22-24], but the reported values differ between these groups [25]. Even a small change in the $\mathrm{Hb}$ and $\mathrm{HbO}_{2}$ extinction coefficients values can cause significant change in the $\mathrm{SaO}_{2}$ calculation when using Equation (4), since the latter includes differences between $\mathrm{Hb}$ and $\mathrm{HbO}_{2}$ extinction coefficients values in the infrared region, where these differences are small relative to those in the red region (Figure 1). 
Figure 7. The extrapolated values of $I_{D}$, and the determination of the pulse amplitude as the difference between the extrapolated values of $I_{D}$ and the measured value of $I_{S}$.

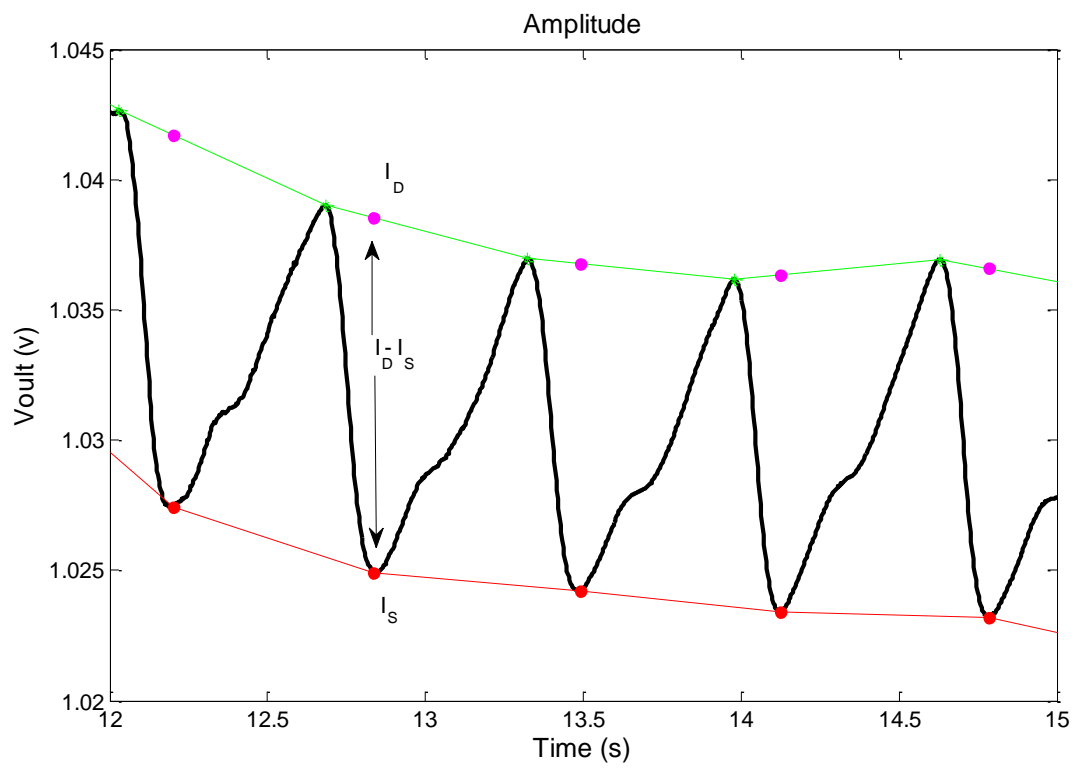

Tables of $\mathrm{Hb}$ and $\mathrm{HbO}_{2}$ extinction coefficients values are available from three research groups (Zijlstra [22], Cope [23] and Prahl [24]), and were also presented for analysis by Kim and Liu [25]. Figure 8 shows the extinction coefficients of $\mathrm{Hb}$ and $\mathrm{HbO}_{2}$ in the near infrared region of 750-850 nm as determined by the three groups. The data presented by Zijlstra in the region above $800 \mathrm{~nm}$ include only two points, at 805 and $840 \mathrm{~nm}$, and interpolation was performed to determine values of the $\mathrm{Hb}$ and $\mathrm{HbO}_{2}$ extinction coefficients for $808 \mathrm{~nm}$.

Figure 8. The extinction coefficients of $\mathrm{Hb}$ and $\mathrm{HbO}_{2}$ in the near infrared region as obtained by Zijlstra et al. (red), Cope (blue) and Prahl (Black) [25]. The red lines represent values derived from the experimental measurements of Zijlstra by means of interpolation (using the pchip command in MATLAB).

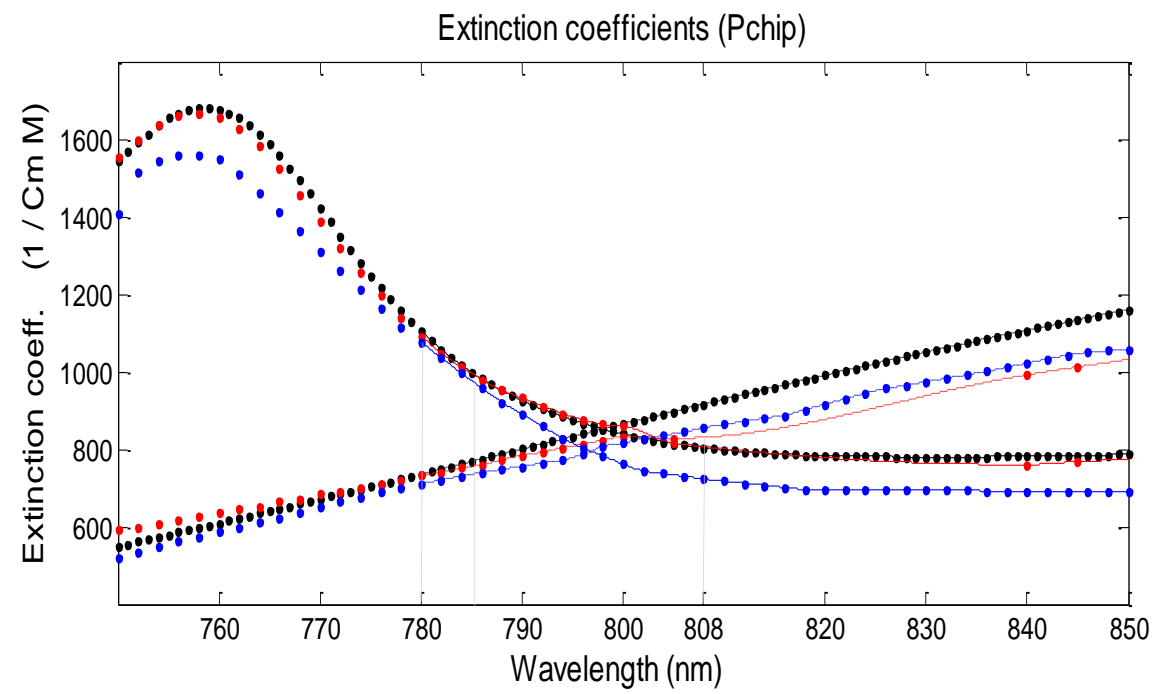


Table 1 presents the extinction coefficients for $\mathrm{HB}$ and $\mathrm{HbO}_{2}$ as derived from the tables of Zijlstra et al., Cope and Prahl, for the three wavelengths which were used for the determination of $\mathrm{SaO}_{2}$ in the current study.

Table 1. The extinction coefficients for $\mathrm{HbO}_{2}$ and $\mathrm{Hb}, \varepsilon_{\mathrm{o}}$ and $\varepsilon_{\mathrm{d}}$, respectively, as derived from the tables of Zijlstra et al, Cope and Prahl.

\begin{tabular}{cccc}
\hline \multirow{2}{*}{ Wavelength $[\mathbf{n m}]$} & \multicolumn{3}{c}{$\boldsymbol{\varepsilon}_{\mathbf{0}} / \boldsymbol{\varepsilon}_{\mathbf{d}}[\mathbf{1} / \mathbf{c m ~ M}]$} \\
\cline { 2 - 4 } & Cope & Prahl & Zijlstra \\
\hline 780 & $738 / 1,098$ & $712 / 1070$ & $733 / 1085$ \\
785 & $769 / 994$ & $736 / 973$ & $757 / 992$ \\
808 & $918 / 803$ & $857 / 723$ & $831 / 806$ \\
\hline
\end{tabular}

\section{Results and Discussion}

\subsection{Results}

PPG recordings for the three wavelengths, 780, 785 and $808 \mathrm{~nm}$ were performed on each of 15 healthy male volunteers. For each pair of wavelengths, the parameter $\mathrm{R}$ was calculated from the corresponding signals (using Equation (5)) and $\mathrm{SaO}_{2}$ was calculated using Equation (4), by making use of the corresponding values of the extinction coefficient from Table 1. In order to validate that the subjects had normal value of oxygen saturation, simultaneous measurements of $\mathrm{SpO}_{2}$ were made on them by means of the commercial pulse oximeter.

Figure 9a shows $\mathrm{SpO}_{2}$ values as obtained for the pair 780-808 nm. The $\mathrm{SpO}_{2}$ values which were determined through the extinction coefficient values obtained by Zijlstra were between 95.3\%-100.5\%. These $\mathrm{SpO}_{2}$ values were similar to those obtained by the commercial pulse oximeter; the difference between them for each examinee was $2.5 \%$ or less (See Table 2). For this pair of wavelengths, $\mathrm{SpO}_{2}$ values determined through the extinction coefficient data obtained by Prahl and Cope were between $86.8 \%-91.0 \%$ and $80.2 \%-84.7 \%$, respectively.

Figure $9 \mathrm{~b}$ shows $\mathrm{SpO}_{2}$ values as obtained for the pair 785-808 nm. For this pair of wavelengths $\mathrm{SpO}_{2}$ values determined through the extinction coefficient values obtained by Zijlstra were between 97.5\%-106.4\%. For the extinction coefficient values obtained by Prahl and Cope $\mathrm{SpO}_{2}$ values were in the range $85.8 \%-92.4 \%$ and $77.6 \%-85.1 \%$, respectively. Figure $9 \mathrm{c}$ shows $\mathrm{SpO}_{2}$ values as obtained for the 780-785 nm pair. For this pair of wavelengths $\mathrm{SpO}_{2}$ values determined by using the extinction coefficient values obtained by Zijlstra were between $81.2 \%-99.7 \%$ and for those obtained by Prahl and Cope were $0.5 \%-3.8 \%$ and $3.3 \%-5.4 \%$ lower, respectively.

The difference between the $\mathrm{SpO}_{2}$ values obtained for the three extinction coefficient databases was small for the wavelengths pair 780-785 nm (Figure 9c), relative to the other wavelengths pairs, because the difference in the extinction coefficient values between the three databases is relatively small for the wavelengths 780 and $785 \mathrm{~nm}$ while for $808 \mathrm{~nm}$ is larger (see Figure 8). Nevertheless, the small difference between the two wavelengths 780 and $785 \mathrm{~nm}$ relative to the other pairs of wavelengths and the fact that they lie on the same side of the isosbestic point lead to a large error in the determination of $\mathrm{R}$ and consequently of $\mathrm{SpO}_{2}$. Since $\mathrm{R}$ is quite close to 1 ( $\mathrm{R}$ is equal to 1 for two equal wavelengths) and the values of the extinction coefficients for oxy- and deoxyhemoglobin are close, the 
values of the numerator and the denominator in Equation (4) are small, and a small error in the determination of $\mathrm{R}$ can cause a large error in the calculated value of $\mathrm{SpO}_{2}$.

Table 2. $\mathrm{SpO}_{2}$ values, obtained by the calibration-free pulse oximetry (based on the $780-808 \mathrm{~nm}$ wavelength pair and on the Zijlstra data) and the commercial pulse oximeter for the 15 healthy subjects.

\begin{tabular}{ccc}
\hline$\#$ & $\begin{array}{c}\text { Calibration-Free } \\
(\boldsymbol{\%})\end{array}$ & $\begin{array}{c}\text { Commercial } \\
(\boldsymbol{\%})\end{array}$ \\
\hline 1 & 98.1 & 97 \\
2 & 97.6 & 96 \\
3 & 96.7 & 98 \\
4 & 100.5 & 98 \\
5 & 98.6 & 97 \\
6 & 95.4 & 96 \\
7 & 95.4 & 97 \\
8 & 98.9 & 97 \\
9 & 98.2 & 97 \\
10 & 95.3 & 96 \\
11 & 98.4 & 97 \\
12 & 99.3 & 98 \\
13 & 98.9 & 97 \\
14 & 98.3 & 97 \\
15 & 95.9 & 96 \\
\hline
\end{tabular}

Figure 9. $\mathrm{SpO}_{2}$ values for the three pairs of wavelengths, determined by using the extinction coefficient data obtained by Zijlstra ( red) Prahl ( black) and Cope (blue).

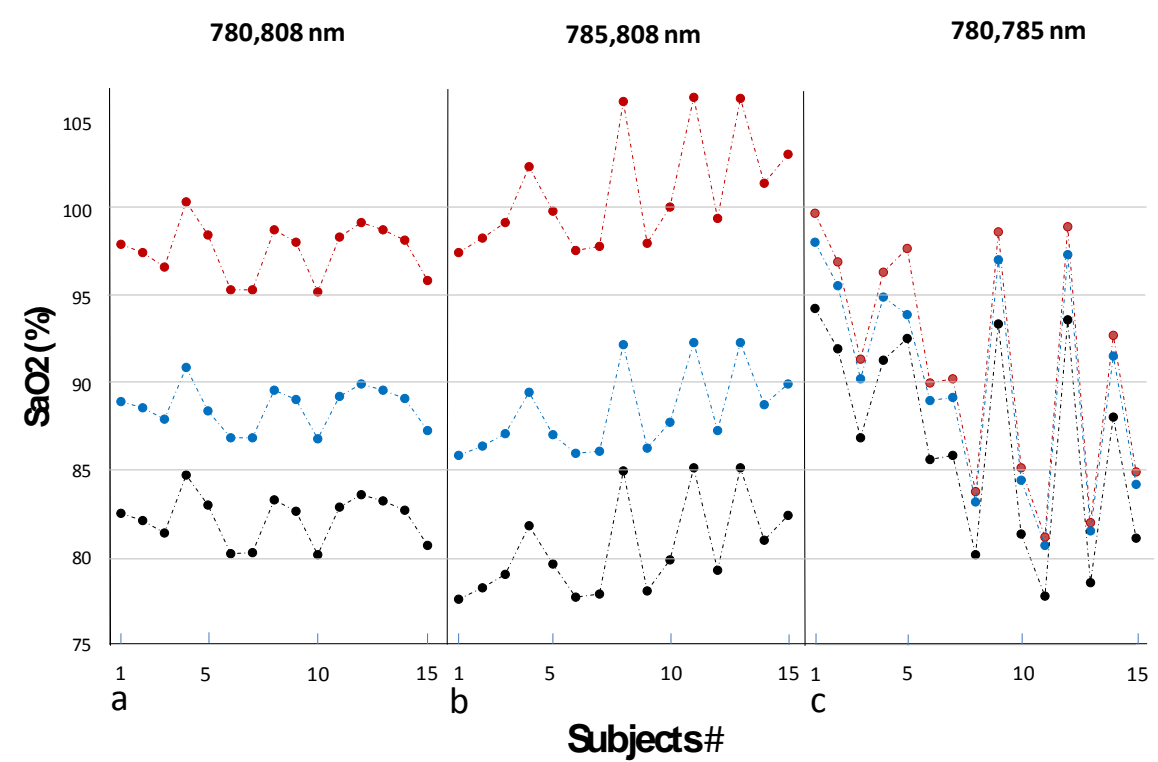




\subsection{Discussion}

In the current preliminary study, we present a calibration-free pulse oximeter that makes use of two nearby wavelengths in the infrared region, assuming that the difference between their path-lengths can be neglected. In this case, by using the published data that gives the relevant extinction coefficients, $\mathrm{SaO}_{2}$ can be derived directly from Equation (4) without calibration. When the extinction coefficients were taken from the data of $\mathrm{Zijlstra}, \mathrm{SpO}_{2}$ measurements, obtained by the calibration-free pulse oximeter, provided values in the normal range of oxygen saturation. It should be emphasized that we did not show that pulse oximetry which uses two wavelengths in the infrared is more accurate than conventional pulse oximetry; we did show that the former can measure $\mathrm{SaO}_{2}$ values in the normal range with reasonable accuracy. While the calibration process cannot decrease the error of conventional pulse oximetry to a level below 3\%-4\%, pulse oximetry with no need for calibration may have the potential to provide more accurate measurements of $\mathrm{SaO}_{2}$ than those provided by the currently available technique.

The proposed technique has several limitations:

(1). The small difference between the two wavelengths makes the values of the extinction coefficients quite close and causes the calculation of $\mathrm{SpO}_{2}$ to be very sensitive to the measured value of $\mathrm{R}$. In order to measure $\mathrm{SpO}_{2}$ accurately, a very accurate measurement of the PPG pulse amplitude is required.

In the current study three pairs of wavelengths were used, and only one of them, the $780-808 \mathrm{~nm}$ pair provided $\mathrm{SpO}_{2}$ results in the required range of oxygen saturation (when Zijlstra extinction coefficients values were used). The results of the 785-808 nm pair were of lower accuracy and the 780-785 nm pair showed the least accuracy. The correlation between the wavelength pair difference and the error in $\mathrm{SpO}_{2}$ measurement can be attributed to the greater sensitivity of $\mathrm{SpO}_{2}$ measurement to the accuracy of $\mathrm{R}$ measurement when two wavelengths with smaller difference between them are used.

(2). There are several databases in which values of the extinction coefficients for oxy- and deoxyhemoglobin have been reported, and there are significant discrepancies between the values obtained in the different studies. It seems that the main problem with the accurate determination of the extinction coefficients for oxy- and deoxyhemoglobin is the measurement of light attenuation in hemolized blood (hemolized blood is used in order to avoid the scattering of light by the red blood cells). The biochemical process of hemolization and the removal of the cells' remains probably interfere with the measurement of light absorption by the hemoglobin molecules. In the current study $\mathrm{SpO}_{2}$ results that lie within the normal range of oxygen saturation values were found only when the extinction coefficients values were selected from the data of Zijlstra; the use of Cope and Prahl data resulted in clearly erroneous values of $\mathrm{SpO}_{2}$. The accuracy of the calibration-free pulse oximetry depends on the accuracy of the extinction coefficients values, and additional research is required in order to find accurate values of the hemoglobin extinction coefficients.

The relative accuracy of the conventional and the calibration-free pulse oximetry should be determined through comparison with invasive in vitro measurements of $\mathrm{SaO}_{2}$. If the novel 
calibration-free pulse oximetry is found to be more accurate than the conventional method, it should have additional diagnostic value in several clinical applications.

It should be noted that some other approaches on calibration-free $\mathrm{SpO}_{2}$ measurement have been proposed in the literature. Reddy et al. [26] suggested a method based on a mathematical model for the attenuation of light passing through the finger. According to their method $\mathrm{SpO}_{2}$ is derived from the amplitudes and slopes of the PPG waveforms acquired at red and infrared excitation wavelengths and the extinction coefficients for $\mathrm{Hb}$ and $\mathrm{HbO}_{2}$. Preliminary examinations performed on healthy volunteers and patients showed agreement with a commercial pulse oximeter. Another calibration-free method based on frequency-modulated near infrared spectroscopy (NIRS) was suggested by Franceschini et al. [27]. However both techniques are based on mathematical models that match the tissue circulation only in approximate terms.

\section{Conclusions}

The pulse oximetry technique for the measurement of oxygen saturation in arterial blood has been improved significantly since its emergence about 30 years ago, but it is still not accurate to better than three or four percent. This inaccuracy is due, at least in part, to the calibration process used, while this process is essential when two significantly different wavelengths are used. The great success of pulse oximeters in detecting significant decrease of oxygen delivery and deterioration of respiratory function in patients during surgical procedures and during recovery probably masks the fact that the accuracy of the present technique is not sufficient to allow its reliable use in certain medical problems, in particular $\mathrm{SaO}_{2}$ monitoring during oxygen supplementation for critically ill patients. Pulse oximetry which uses two nearby wavelengths is a greater technological challenge, but since it does not require initial calibration it has the potential to provide the clinical community with more accurate arterial oxygen saturation values than those obtained by the available pulse oximeters which use two wavelengths in the red and infrared regions and need calibration.

\section{Acknowledgments}

The study was supported by Joseph Trust, UK.

\section{Author Contributions}

Conception and design of the study: M. Nitzan. Design of the pulse oximeter and technical support: S. Noach, E. Tobal, Y. Adar, Y. Miller, E. Shalom. Collection of data: E. Tobal, Y. Adar, Y. Miller. Analysis and interpretation of the data: M. Nitzan, S. Engelberg, E. Tobal, Y. Adar. Manuscript preparation: M. Nitzan. Critical revision of the article: S. Engelberg.

\section{Conflicts of Interest}

The authors declare no conflict of interest. 


\section{References}

1. O’Driscoll, B.R.; Howard, L.S.; Davison A.G. on behalf of the British Thoracic Society. BTS guideline for emergency oxygen use in adult patients. Thorax 2008, 63 (Suppl 6), vi1-vi68.

2. Yoshiya, I.; Shimady, Y.; Tanake, K. Spectrophotometric monitoring of arterial oxygen saturation on the fingertip. Med. Biol. Eng. Comput. 1980, 18, 27-32.

3. Wieben, O. Light absorbance in pulse oximetry. In Design of Pulse Oximeters; Institute of Physics Publishing: Bristol, UK, 1997; pp. 40-55.

4. Moyle, J.T.B. Pulse Oximetry; BMJ Books: London, UK, 2002.

5. Mannheimer, P.D. The Light-Tissue Interaction of Pulse Oximetry. Anesth. Analg. 2007, 105, S10-S17.

6. Sebald, D.J. Motivation in pulse oximetry. In Design of Pulse Oximeters; Institute of Physics Publishing: Bristol, UK, 1997; pp. 13-20.

7. Prahl, S. The absorption spectra of the oxygenated and deoxygenated hemoglobin molecules. graph. Available online: http://omlc.ogi.edu/spectra/hemoglobin/index.html (accessed on 21 January 2014).

8. Mannheimer, P.D.; Casciani, J.R.; Fein M.E.; Nierlich, S.L. Wavelength selection for low-saturation pulse oximetry. IEEE Trans. Biomed. Eng. 1997, 44, 148-158.

9. Zourabian, A.; Siegel, A.; Chance, B.; Ramanujan, N.; Rode, M.; Boas, D.A. Trans-abdominal monitoring of fetal arterial blood oxygenation using pulse oximetry. J. Biomed. Opt. 2000, 5, 391-405.

10. Kyriacou, P.A. Pulse oximetry in the esophagus. Physiol. Meas. 2006, 27, R1-R35.

11. Nitzan, M.; Engelberg, S. Three-wavelength technique for the measurement of oxygen saturation in aterial blood and in venous blood. J. Biomed. Opt. 2009, 14, 024046.

12. Friebel, M.; Helfmann, J.; Netz, U.; Meinke, M. Influence of oxygen saturation on the optical scattering properties of human red blood cells in the spectral range 250 to $2000 \mathrm{~nm}$. J. Biomed. Opt. 2009, 14, 034001.

13. Batchelder, P.B.; Raley, D.M. Maximizing the laboratory setting for testing devices and understanding statistical output in pulse oximetry. Anest. Analg. 2007, 105, S85-S94.

14. Fouzas, S.; Priftis K.N.; Anthrcopoulos, M.B. Pulse oximetry in pediatric practice. Pediatrics 2011, 128, 740-752.

15. Seguin, P.; Le Rouzo, A.; Tanguy, M.; Guillou, Y.M.; Feuillu, A.; Malledant, Y. Evidence for the need of bedside accuracy of pulse oximetry in an intensive care unit. Crit. Care Med. 2000, 28, 703-706.

16. Van de Louw, A.; Cracco, C.; Cerf, C.; Harf, A.; Duvaldestin P.; Lamaire P,F.; Brochard, L. Accuracy of pulse oximetry in the intensive care unit. Intens. Care Med. 2001, 27, 1606-1613.

17. Perkins, G.D.; McAuley, D.F.; Giles, S.; Routledge, H.; Gao, F. Do changes in pulse oximeter oxygen saturation predict equivalent changes in arterial oxygen saturation? Crit. Care 2003, 7 , R67-R71.

18. Wilson, B.J.; Cowan, H.J.; Lord, J.A.; Zuege, D.J.; Zygun, D.A. The accuracy of pulse oximetry in emergency department patients with severe sepsis and septic shock: A retrospective cohort study. BMC Emerg. Med. 2010, 10, 9. 
19. Poets, C.F.; Southall, D.P. Noninvasive monitoring of oxygenation in infants and children: practical considerations and areas of concern. Pediatrics 1994, 93, 737-746.

20. Tungjitkusolmun, S. Accuracy and errors. In Design of Pulse Oximeters; Institute of Physics Publishing: Bristol, UK, 1997; pp. 176-198.

21. Nitzan, M.; Babchenko, A.; Khanokh, B.; Taitelbaum, H. The measurement of oxygen saturation in venous blood by dynamic near IR spectroscopy. J. Biomed. Opt. 2000, 5, 155-162.

22. Zijlstra, W.G.; Buursma, A.; Meeuwsen-van der Roest, W.P. Absorption spectra of human fetal and adult oxyhemoglobin, de-oxyhemoglobin, carboxyhemoglobin, and methemoglobin. Clin. Chem. 1991, 37, 1633-1638.

23. Cope, M. The application of near infrared spectroscopy to non invasive monitoring of cerebral oxygenation in the newborn infant. Ph.D. Thesis, University College London: London, UK, 1991.

24. Prahl, S.A. Tabulated Molar Extinction Coefficient for Hemoglobin in Water. Available online: http://omlc.ogi.edu/spectra/hemoglobin/summary.hmtl (accessed on 14 January 2014).

25. Kim J.G.; Liu, H. Variation of haemoglobin extinction coefficients can cause errors in the determination of haemoglobin concentration measured by near-infrared spectroscopy. Phys. Med. Biol. 2007, 52, 6295-6322.

26. Reddy, K.A.; George, B.; Mohan, N.N.; Kumar, V.J. A novel calibration-free method of measurement of oxygen saturation in arterial blood. IEEE Tr. BME 2009, 58, 1699-1705.

27. Franceschini, M.A.; Gratton, E.; Fantini, S. Noninvasive optical method of measuring tissue and arterial saturation: An application to absolute pulse oximetry of the brain. Opt. Lett. 1999, 24, 829-831.

(C) 2014 by the authors; licensee MDPI, Basel, Switzerland. This article is an open access article distributed under the terms and conditions of the Creative Commons Attribution license (http://creativecommons.org/licenses/by/3.0/). 